\title{
Critical appraisal of
}

volumetric-modulated arc

\section{therapy compared with}

electrons for the

\section{radiotherapy of cutaneous}

\section{Kaposi's sarcoma of lower extremities with bone sparing}

\author{
'G NICOLINI, MSc, ${ }^{2} S$ ABRAHAM, MD, 'A FOGLIATA, MSc, ${ }^{2}$ A JORDAAN, MD, 'A CLIVIO, MSc, \\ 'E VANETTI, MSC and 'L COZZI, PhD
}

'Medical Physics Unit, Radiotherapy Department, Oncology Institute of Southern Switzerland, Bellinzona, Switzerland

${ }^{2}$ Department of Radiation Oncology, Addington Hospital, Durban, South Africa

Address correspondence to: Mrs Giorgia Nicolini

E-mail: giorgia.nicolini@eoc.ch

Objective: To evaluate the use of volumetricmodulated arc therapy [VMAT, RapidArc ${ }^{\circledR}$ (RA); Varian Medical Systems, Palo Alto, CA] for the treatment of cutaneous Kaposi's sarcoma (KS) of lower extremities with adequate target coverage and high bone sparing, and to compare VMAT with electron beam therapy.

Methods: 10 patients were planned with either RA or electron beams. The dose was prescribed to $30 \mathrm{~Gy}, 10$ fractions, to mean the planning target volume (PTV), and significant maximum dose to bone was limited to $30 \mathrm{~Gy}$. Plans were designed for 6-MV photon beams for RA and $6 \mathrm{MeV}$ for electrons. Dose distributions were computed with $A$ curos $X B^{\circledR}$ (Varian Medical Systems) for photons and with a Monte Carlo algorithm for electrons.

Results: $V_{90 \%}$ was $97.3 \pm 1.2$ for RA plans and $78.2 \pm 2.6$ for electrons; similarly, $V_{107 \%}$ was $2.5 \pm 2.2$ and $37.7 \pm 3.4$, respectively. RA met coverage criteria. Concerning bone sparing, $D_{2 \%}$ was $29.6 \pm 1.1$ for $R A$ and $31.0 \pm 2.4$ for electrons. Although acceptable for bone involvement, pronounced target coverage violations were obtained for electron plans. Monitor units were similar for electrons and RA, although for the latter they increased 
when superior bone sparing was imposed. Delivery times were $12.1 \pm 4.0 \mathrm{~min}$ for electrons and $4.8 \pm 1.3 \mathrm{~min}$ for the most modulated RA plans.

Conclusion: High plan quality was shown for $\mathrm{KS}$ in the lower extremities using VMAT, and this might simplify their management in comparison with the more conventional usage of electrons, particularly in institutes with limited staff resources and heavy workloads.

Advances in knowledge: VMAT is also dosimetrically extremely advantageous in a typology of treatments where electron beam therapy is mainly considered to be effective owing to the limited penetration of the beams.
Kaposi's sarcoma (KS) is frequently demonstrated in patients infected by human immunodeficiency virus (HIV)([1], epidemic form) and can be manifested in the mouth, lymphatic system, gastro-intestinal system, lungs and skin. KS can be considered as generally responsive to radiation therapy, and good palliative (and cosmetic) results were demonstrated with doses higher than $20 \mathrm{~Gy}$ [2-4]. In 1996, Stein et al [5] demonstrated that, in South Africa, all types of KS were present and that response rates as high as $90 \%$ were achieved for the epidemic form. In addition, the study proved that radiation therapy allowed better symptomatic control than chemotherapy. The randomised trial of Singh et al [4] demonstrated the feasibility of hypofractionation in terms of recurrence-free survival, toxicity and local control. Even a single fraction of $8 \mathrm{~Gy}[6,7]$ showed to be feasible in patients with limited median life expectancy. Conill et al [8] suggested that the range from $8 \mathrm{~Gy}$ in a single fraction to $30 \mathrm{~Gy}$ in ten fractions, tailored to individual patient needs, permits the achievement of local control with minimal toxicity. Although conventional photon therapy can be applied to most of the cases, for cutaneous lesions, electron beam therapy is considered to be effective because of the limited penetration of the beams, and it would also allow the risk of radiation-induced oedema of the legs as a consequence of reduced lymphatic drainage to be minimised. Geara et al [9] showed that, with electrons of 4-8 MeV, the main toxicity was dry epidermitis with limited frequency of skin ulcerations. The obvious limit of electrons is that when extensive KS is treated, the large number of treatment fields necessary to cover the target and the complex positioning of the same lead to very long treatment times and risk of under- or overirradiation of the skin (when field matching is needed and because of the convexity of the target), requiring very careful patient positioning procedures. In busy clinics with heavy patient loads, the usage of electrons for cutaneous KS could therefore be problematic for a smooth workflow.
RapidArc ${ }^{\circledR}$ (RA; Varian Medical Systems, Palo Alto, CA) is a relatively new volumetric-modulated arc therapy (VMAT) technique based on simultaneous optimisation of the multileaf collimator (MLC) shape, dose rate and gantry rotation speed [10]. The technology was investigated in several studies, showing a general improvement in organs at risk and healthy tissue sparing, similarly good target coverage, reduced beam-on time and lower number of monitor units (MUs) compared with other intensity-modulated radiotherapy approaches with fixed gantry entrances. Many indications can benefit from the RA technology, and, regarding sarcomas, little has been published to date. LlacerMoscardo et al [11] investigated the potential of this VMAT approach for huge retroperitoneal sarcoma, this being the seminal study for the application of the new treatment technique to this class of tumours. A more recent study [12] assessed the positive role of RA in the case of soft-tissue sarcoma in the legs with the possibility of significantly sparing the femurs. Given the anatomical analogy, a similar planning approach was mimicked in the present study, with the main difference given by the fact that, here, target volumes are extended over the entire lower legs but are limited to a relatively superficial layer. In this context, the role of RA for superficial targets has not been extensively studied. An investigation [13] demonstrated that, with the first generation of the optimisation engine, it was possible to achieve the desired target homogeneity using multiple arcs. In that study, the clinical case (a paediatric brain tumour) was quite different from the problem under investigation here, but it was encouraging as a proof of principle.

The aim of this work is to present a comparative planning study on a group of patients to be treated for cutaneous KS of the lower extremities, comparing dose distributions achieved with RA against the more conventional 
approach based on electron fields. The planning challenge was to achieve a high degree of bone and normal tissue sparing to minimise the risk of fractures and other disease-related morbidities, which prevented the use of simple and straightforward techniques such as opposed photon fields. In addition to dosimetric plan quality, treatment efficiency was also scored. This was mostly determined by treatment time, and was assessed to clarify the possibility of using a high technology treatment modality like RA in institutes with limited staff resources and heavy patient load, as in Africa.

\section{MATERIALS AND METHODS}

A total of 10 patients eligible for radiation treatment of cutaneous KS in the lower legs were included in the present planning study. 7 out of 10 patients presented with superficial target volumes adjacent to the leg's bones and extending from the knees to the base of the foot. The limited number of patients is in line with consolidated practice of planning studies, while the inclusion of the foot in seven patients added extra interpatient variability in the target definition and therefore contributed to increased robustness of results and the potential of generalisation. Target volume longitudinal length was on average $45 \pm 12.0 \mathrm{~cm}$ (range $28.5-66.0 \mathrm{~cm}$ ).

Planning CT (Siemens Somatom Sensation; Siemens AG, Munich, Germany) was acquired with adjacent 3-mm slices. Patient positioning was supine with immobilisation of the leg using a customised vacuum cushion to ensure daily reproducibility. The Eclipse treatment planning system v. 11 (Varian Medical Systems) was used for contouring, planning and dose calculation.

The planning target volume (PTV) was defined according to the institutional practice from the skin surface to a constant depth of $1 \mathrm{~cm}$ along the entire leg portion presenting lesions. From the PTV, the bones were subtracted.

In the absence of an absolute consensus for high hypofractionation, a moderate hypofractionation schedule was assumed with a dose prescription of $30 \mathrm{~Gy}$ in 10 fractions. All RA plans were normalised individually to mean the PTV dose according to the International Commission on Radiation Units and Measurements report ICRU 83 [14] specifications for intensity-modulated fields. For comparison reasons, the same strategy was adopted for electron plans. Computed MUs were recorded for each plan.
Given the complex target shape, particularly when the base of foot was included, plans were designed and optimised to achieve a relative relaxed PTV coverage of $V_{90 \%}>95 \%$ and $V_{107 \%}<2 \%$. To control the involvement of the bones, the objective was to keep the significant maximum dose below the prescription dose of $30 \mathrm{~Gy}$.

\section{Photon RA plans}

Three plans per patient were prepared: photon RA plans (RA_1 and RA_2) and electron plans. The photon RA plans involved two to four partial arcs, avoiding beam entrance from the contralateral leg, and collimator rotation near or equal to $10^{\circ}$ to better spare the bones. When PTV longitudinal length required it, two isocenters (of two arcs each) were used with an overlapping region of at least $2 \mathrm{~cm}$ between upper and lower arcs [15]. Figure 1 illustrates, for one arc, the target and the field as defined by the jaws (solid external line) and the MLC aperture (at one control point). Given the

Figure 1. Exemplification of the beam setting for the RapidArc ${ }^{\circledast}$ (Varian Medical Systems, Palo Alto, CA) plans.

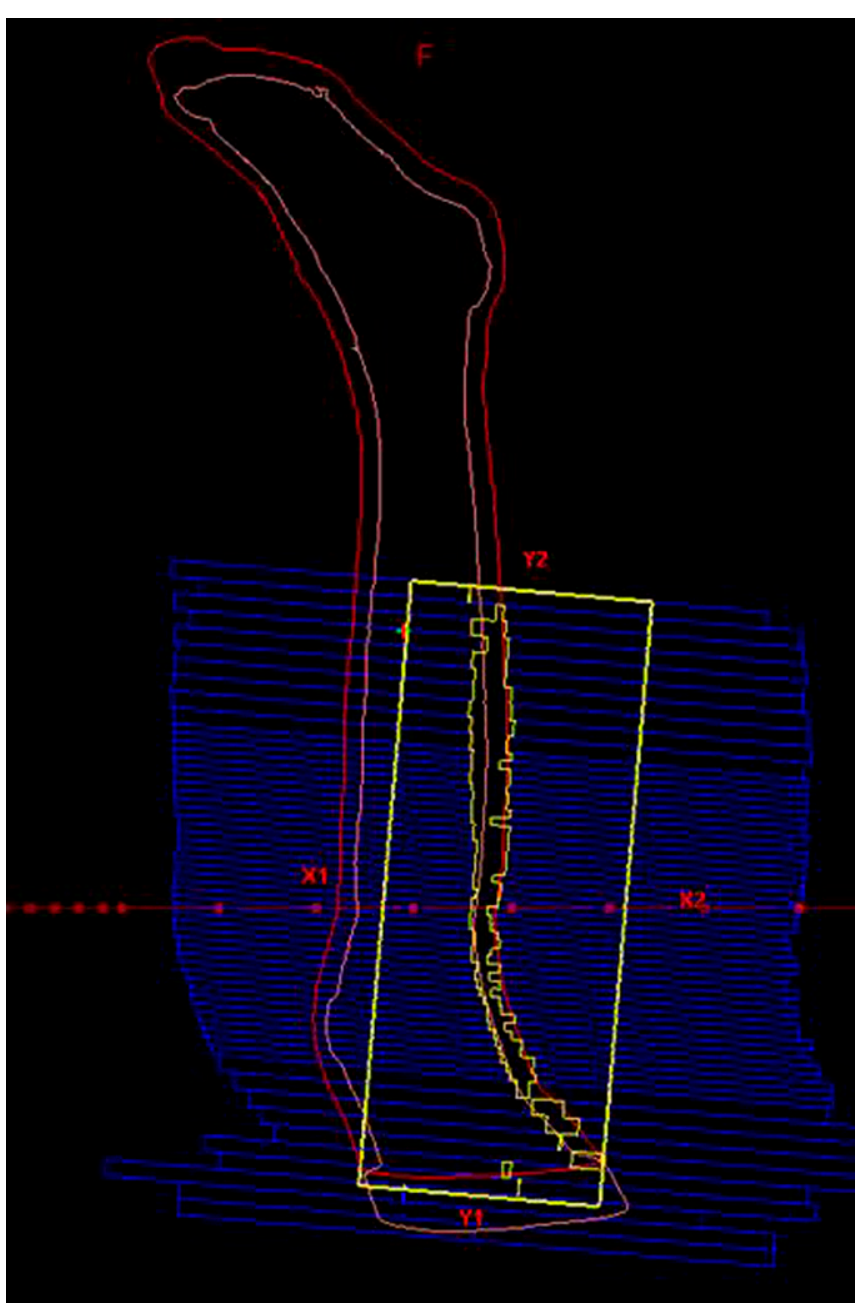


specificity of the PTV, asymmetric jaws setting was chosen in order to "cover" only one side of the target at the time to maximise the sparing of the inner leg tissue. A bolus of $6 \mathrm{~mm}$ was defined and customised for each patient using the automatic bolus tool of Eclipse (in the case of real treatment, a natural wax bolus would have been prepared for each individual and CT scans would have been acquired with the bolus). Two groups of plans were generated: the first group, RA_1, had the aim of achieving planning objectives for target coverage, homogeneity and maximum dose to the bones; the second group, RA_2, was generated adding the request to maximise bone sparing without significant compromises to target objectives. Both groups adopted the same beam arrangements and differed only for the optimisation criteria.

Plans were optimised with the Progressive Resolution Optimizer algorithm PRO3 (Varian Medical Systems) [16]. A Varian Clinac 2100iX equipped with a Millennium MLC-120 (Varian Medical Systems) was used with the selection of 6-MV photon beams. Final dose calculations were performed with a grid size of $2.5 \mathrm{~mm}$, with the Acuros $\mathrm{XB}^{\circledR}$ Advanced Dose Calculation (Varian Medical Systems) [17], v. 11.0.21, using the dose-tomedium option.

\section{Electron plans}

A variable number of electron fields were defined to cover the target, this ranged from 6 to 18 . A $6 \mathrm{MeV}$ electron beam from a Varian Clinac 2100iX was used for the study. For shorter targets, the leg was covered with six fields (gantry angles $0^{\circ}, 60^{\circ}, 120^{\circ}, 180^{\circ}, 240^{\circ}$ and $300^{\circ}$ ). For longer targets, as for the RA case with multiple isocenters, a second couch position was adopted (changing the $z$ co-ordinate) for a second group of six fields with the same gantry angles. To minimise the number of needed $z$ positions, sourceskin distance was increased from 100 to $130 \mathrm{~cm}$ for some patients, resulting in a $32 \times 32 \mathrm{~cm}^{2}$ nominal field. A third couch position, with $90^{\circ}$ pedestal rotation, was used for one field in three patients to allow coverage of the base of the foot. For all fields, a $25 \times 25 \mathrm{~cm}^{2}$ applicator was selected and the collimator angle was adjusted individually with a rotation of $\sim 0^{\circ}$ for multiple couch longitudinal positions. A collimator rotation of $\sim 45^{\circ}$ was conversely used for single $z$ position to cover longer volumes. Field matching between upper and lower electron fields was properly adjusted. Individual moulds were defined to shape each of the fields.
Dose calculation was performed within the Eclipse 11 environment using the electron Monte Carlo (eMC) algorithm (v. 11.0.21; Varian Medical Systems) [18, 19] with a calculation grid of $2.5 \mathrm{~mm}$. Accuracy was set to $2 \%$ [defined as the average statistical uncertainty in the maximum dose $\left(D_{\max }\right)$ region] and this parameter was used by the system instead of a pre-defined number of histories to be simulated. A three-dimensional (3D) Gaussian smoothing method was applied to final doses with a "medium" strength, as defined by the planning system.

\section{Evaluation}

Plan evaluation was assessed by dose-volume histograms (DVHs) analysis. For the PTV, the following parameters were studied: volume $x\left(V_{x}\right)$ with $x=90 \%, 95 \%, 107 \%$ and $120 \%, D_{y}$ with $y=2 \%, 5 \%, 95 \%$ and $98 \%\left(D_{2 \%}\right.$ and $D_{98 \%}$ can be seen as maximum and minimum significant doses), $D_{5 \%}-D_{95 \%}$ was defined as an index of dose homogeneity. For bones, the analysed parameters were $D_{2 \%}$ (maximum significant dose), $D_{10 \mathrm{~cm}^{3}}$ and $D_{20 \mathrm{~cm}^{3}}$, and $V_{x}$ with $x=10,20,30 \mathrm{~Gy}$; the value of the mean dose was reported as a measure of the whole-bone irradiation. No other specific organs at risk were analysed, but the normal healthy tissue was defined as the body volume in the CT scan minus the PTV. Average cumulative DVH for PTV, bones and healthy tissues were built from the individual DVHs and presented in the results. These histograms were obtained by averaging the corresponding volumes over the whole patient's cohort for each dose bin of $0.05 \mathrm{~Gy}$.

To evaluate the treatment efficiency, MUs, planning times and delivery times were recorded for each technique. The latter was measured as the time needed to treat a complete plan, inclusive of beam on time and machine reset between different fields or arcs. This included the time needed to enter in the treatment room between each electron field and the time needed for imaging procedures with two-dimensional (2D-2D) matching of orthogonal planar electronic portal. To avoid bias owing to different hardware configurations in other institutes, planning time was expressed in absolute and relative terms as the ratio of the time needed to plan RA $v s$ the time needed to plan electrons. The planning time included beam settings, optimisation time and dose calculation.

Statistical significance of the differences between mean values of the various parameters was investigated by means of the non-parametric Fisher's sign test (the 
standard tests based on $\chi^{2}$ cannot be used because of the small sample size).

\section{RESULTS}

In Figure 2, dose distributions (colour wash up to $36 \mathrm{~Gy}$ ) for one example patient for the electron-based plan and the two RA plans are shown. Qualitatively, it is visible how the RA plans allow for more homogeneity while dose distribution relative to electrons is characterised by relevant cold and hot spots. Figure 3 represents the average DVHs for PTV and bone and demonstrates qualitatively all the main findings of the study. For the PTV, there is very low target coverage of electrons (as well as significant overdosage) and a modest difference between RA_1 and RA_2. This is an acceptable trade-off if compared with the difference in bone irradiation. In this case, RA_2 allowed a systematic and macroscopic sparing of the bone tissue compared with RA_1 and also a systematic further sparing (at medium to high doses) compared with electrons. Average DVH for bones (and healthy tissue) demonstrates how effectively RA allows modulation of dose inside the leg without a clinically relevant price to pay in terms of target coverage.

Table 1 presents quantitative results of DVH analysis for PTV and bone as well as for the healthy tissue, averaged over the 10 patients.
PTV coverage was similar in and acceptable for both RA_1 and RA_2 plans, although RA_2 presented some minor deviation with respect to the planning objectives. On the contrary, electron plans resulted in severe underand overdosage of the PTV, with an average of $7 \%$ of it receiving $>120 \%$ of the prescribed dose. Homogeneity of irradiation was best with the RA_1 plan, with a significant worsening, although considered of no clinical relevance, for RA_2. Electron plans resulted in a very heterogeneous dose distribution, with a homogeneity of about a factor of 8 worse than RA_1 and a factor of 4 worse than RA_2.

The bone maximum dose, denoted as $D_{2 \%}$, objective of $30 \mathrm{~Gy}$ was met in average only for RA_2 plans; minor deviations were obtained for RA_1 and electron plans. The different planning objectives between the two RA groups allowed the significant reduction of bone involvement over the entire dose range (above $5 \mathrm{~Gy}$ ) and also the improvement of it with respect to electrons above a threshold of about $8 \mathrm{~Gy}$. Healthy tissue involvement reflected the same trend as for the bone with RA_2-the technique allowing the better sparing.

Statistically significant differences were observed for most of the parameters, as summarised in Table 2.

Figure 2. Isodose distributions for electrons and RapidArc ${ }^{\circledR}$ (Varian Medical Systems, Palo Alto, CA) plans. Colourwash scale extends to 36 Gy.

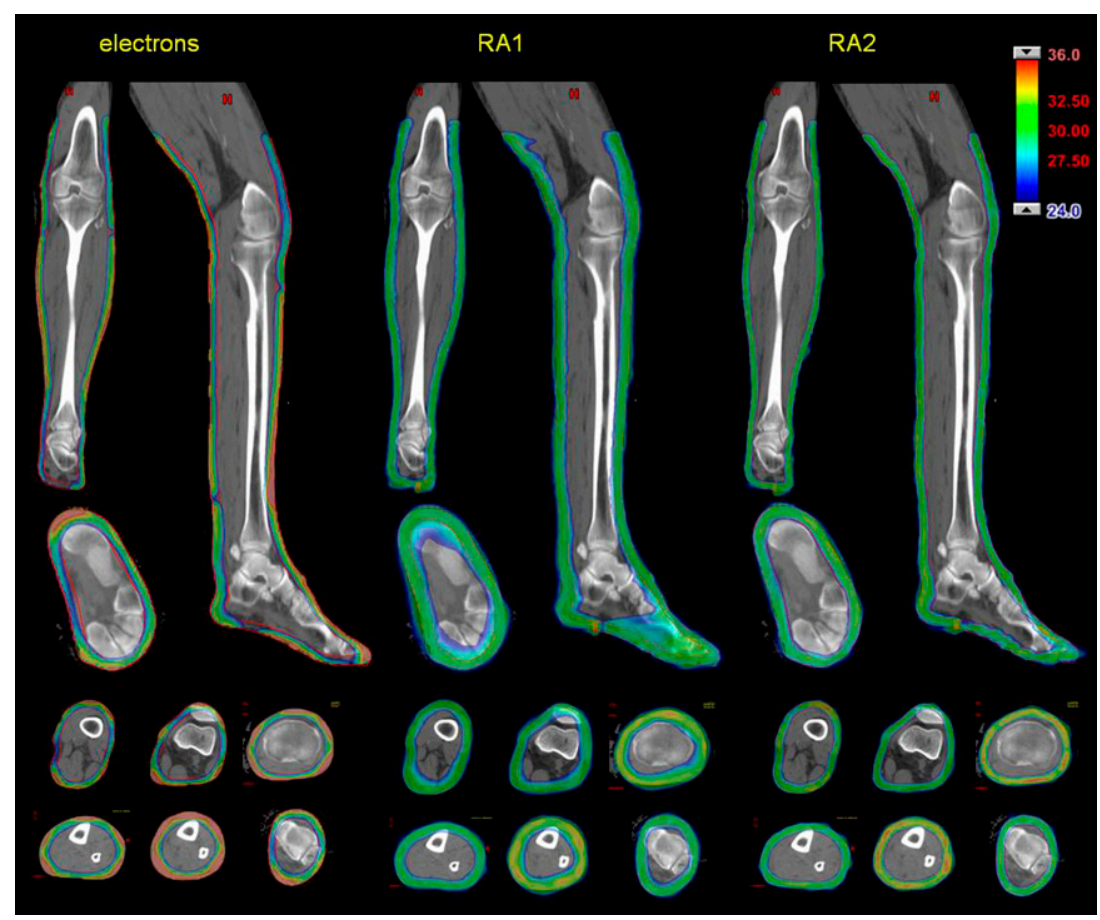


Figure 3. Average dose-volume histograms for planning target volume (PTV), bone and healthy tissue for all techniques.
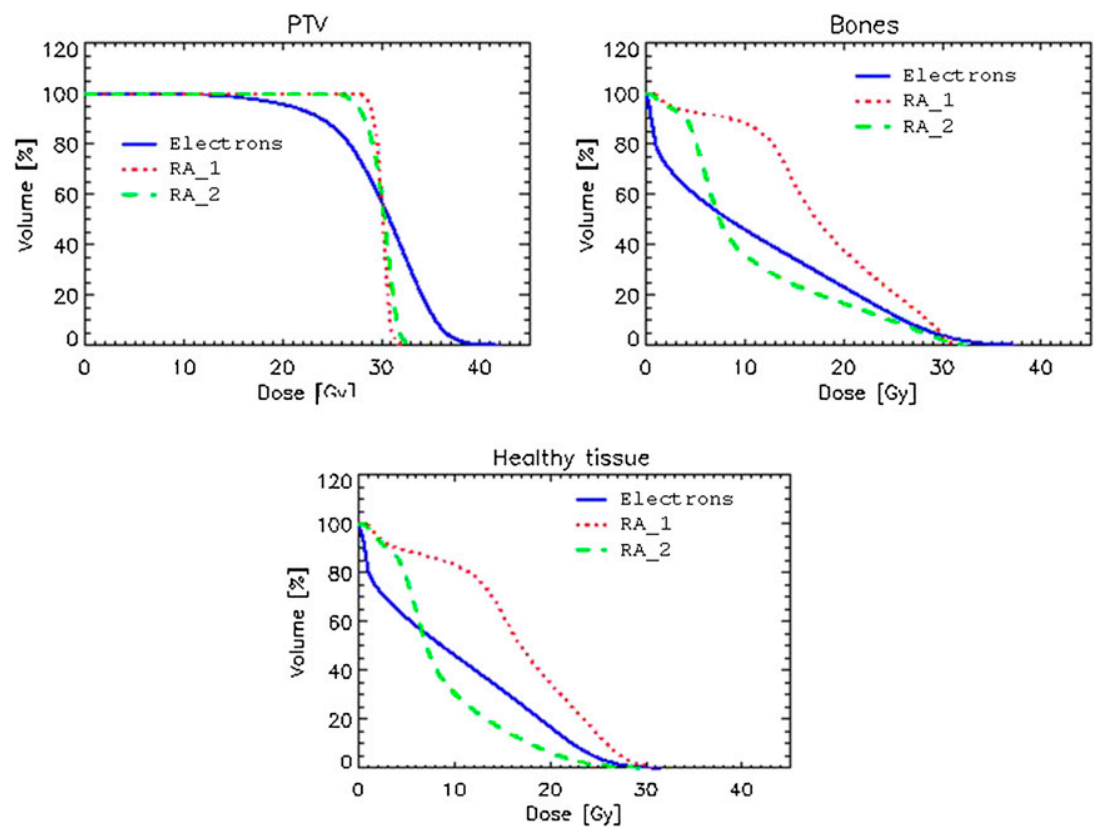

The calculated MUs for electrons were $1630 \pm 436$ with a range of 952-2437. MUs for RA_1 were 1556 \pm 385 (range: 998-2123) and for RA_2 were 2576士672 (range: 1771-3664) with the higher number for RA_2

than for RA_1 a result of the higher modulation needed for further bone sparing. The MU difference between RA_2 and RA_1 or electrons is statistically significant in both cases.

Table 1. Summary of the main technical details for the electron and RapidArc ${ }^{\circledR}$ (RA) (Varian Medical Systems, Palo Alto, CA) plans

\begin{tabular}{|c|c|c|}
\hline Technical details & Mean \pm SD & Range \\
\hline PTV $\left(\mathrm{cm}^{3}\right)$ & $1177 \pm 388$ & $682-2010$ \\
\hline PTV length (cm) & $45 \pm 12$ & $28.5-66$ \\
\hline Foot inclusion & $7 / 10 \mathrm{pts}$ & \\
\hline \multicolumn{3}{|l|}{ Electrons, eMC algorithm } \\
\hline Energy $(\mathrm{MeV})$ & 6 & - \\
\hline Applicator $(\mathrm{cm} \times \mathrm{cm})$ & $25 \times 25$ & - \\
\hline Source-skin distance $(\mathrm{cm})$ & $112 \pm 10$ & $100-130$ \\
\hline Collimator $\sim 0^{\circ}$ & $7 / 10 \mathrm{pts}$ & - \\
\hline Collimator $\sim 45^{\circ}$ & $3 / 10 \mathrm{pts}$ & - \\
\hline Number of fields & $11 \pm 3.7$ & $6-18$ \\
\hline Field towards foot base & $4 / 7 \mathrm{pts}$ & - \\
\hline Number of $z$ entries & $1.8 \pm 0.6$ & $1-3$ \\
\hline \multicolumn{3}{|l|}{ RA photons } \\
\hline Number of isocenters & $1.7 \pm 0.5$ & $1-2$ \\
\hline Number of arcs & $3.4 \pm 1.0$ & $2-4$ \\
\hline \multicolumn{3}{|l|}{ Field size RA_1 } \\
\hline \multicolumn{3}{|l|}{ Field size RA_2 } \\
\hline Total rotation $\left(^{\circ}\right)$ & $852 \pm 297$ & $264-1104$ \\
\hline
\end{tabular}

eMC, electron Monte Carlo; pts, patients; PTV, planning target volume. 
Table 2. Summary of dose-volume histogram analysis for planning target volume (PTV), bone and healthy tissue for all techniques

\begin{tabular}{|c|c|c|c|c|c|}
\hline \multirow{2}{*}{ Structure } & \multirow{2}{*}{ Parameter } & Electrons & RA_1 & RA_2 & \multirow{2}{*}{$p$} \\
\hline & & \multicolumn{3}{|c|}{ Mean \pm standard deviation } & \\
\hline$P T V$ & Mean (Gy) & $30.0 \pm 0.0$ & $30.0 \pm 0.0$ & $30.0 \pm 0.0$ & \\
\hline \multirow{7}{*}{$\begin{array}{l}\text { Volume }\left(\mathrm{cm}^{3}\right)=1177 \pm 388 \\
\text { Length }(\mathrm{cm})=45.5 \pm 11.8(28.5 ; 66.0)\end{array}$} & $D_{5 \%}-D_{95 \%}(\mathrm{~Gy})$ & $15.9 \pm 2.2$ & $2.1 \pm 0.3$ & $4.3 \pm 0.6$ & $\mathrm{a}, \mathrm{b}, \mathrm{c}$ \\
\hline & $D_{2 \%}(\mathrm{~Gy})$ & $37.6 \pm 1.2$ & $31.2 \pm 0.2$ & $32.1 \pm 0.3$ & a, b, c \\
\hline & $D_{98 \%}(\mathrm{~Gy})$ & $16.9 \pm 2.2$ & $28.5 \pm 0.2$ & $26.8 \pm 0.4$ & $\mathrm{a}, \mathrm{b}, \mathrm{c}$ \\
\hline & $V_{90 \%}(\%)$ & $78.2 \pm 2.6$ & $100.0 \pm 0.0$ & $97.3 \pm 1.2$ & $\mathrm{a}, \mathrm{b}, \mathrm{c}$ \\
\hline & $V_{95 \%}(\%)$ & $68.5 \pm 2.4$ & $97.9 \pm 1.2$ & $87.3 \pm 2.6$ & a, b, c \\
\hline & $V_{107 \%}(\%)$ & $37.7 \pm 3.4$ & $0.1 \pm 0.1$ & $2.5 \pm 2.2$ & $\mathrm{a}, \mathrm{b}, \mathrm{c}$ \\
\hline & $V_{120 \%}(\%)$ & $7.0 \pm 3.5$ & $0.0 \pm 0.0$ & $0.0 \pm 0.0$ & $a, b$ \\
\hline Bone & Mean (Gy) & $10.9 \pm 3.6$ & $17.8 \pm 2.8$ & $10.7 \pm 2.3$ & a, c \\
\hline \multirow{6}{*}{ Volume $\left(\mathrm{cm}^{3}\right)=597 \pm 225$} & $D_{2 \%}(\mathrm{~Gy})$ & $31.0 \pm 2.4$ & $30.1 \pm 0.7$ & $29.6 \pm 1.1$ & b, c \\
\hline & $V_{10 \mathrm{~Gy}}(\%)$ & $45.7 \pm 15.0$ & $88.0 \pm 12.3$ & $35.7 \pm 12.1$ & $\mathrm{a}, \mathrm{b}, \mathrm{c}$ \\
\hline & $V_{20 \mathrm{~Gy}}(\%)$ & $23.0 \pm 11.6$ & $37.6 \pm 13.7$ & $16.5 \pm 7.6$ & $a, b, c$ \\
\hline & $V_{30 \mathrm{~Gy}}(\%)$ & $3.9 \pm 3.4$ & $3.7 \pm 3.3$ & $2.1 \pm 1.7$ & b, c \\
\hline & $D_{10 \mathrm{cc}}(\mathrm{Gy})$ & $31.4 \pm 2.4$ & $30.2 \pm 0.9$ & $29.7 \pm 1.3$ & b, c \\
\hline & $D_{20 \mathrm{cc}}(\mathrm{Gy})$ & $29.4 \pm 2.6$ & $29.6 \pm 1.2$ & $28.4 \pm 2.0$ & $\mathrm{~b}, \mathrm{c}$ \\
\hline Healthy tissue & Mean (Gy) & $10.0 \pm 1.6$ & $16.6 \pm 1.7$ & $8.8 \pm 0.8$ & $\mathrm{a}, \mathrm{b}, \mathrm{c}$ \\
\hline \multirow{2}{*}{ Volume $\left(\mathrm{cm}^{3}\right)=1894 \pm 737$} & $D_{2 \%}(\mathrm{~Gy})$ & $26.3 \pm 1.5$ & $28.7 \pm 1.1$ & $23.8 \pm 1.3$ & $\mathrm{a}, \mathrm{b}, \mathrm{c}$ \\
\hline & $V_{10 \mathrm{~Gy}}(\%)$ & $46.0 \pm 7.4$ & $83.1 \pm 9.5$ & $30.2 \pm 3.9$ & $\mathrm{a}, \mathrm{b}, \mathrm{c}$ \\
\hline
\end{tabular}

$D_{x \%}$, dose received by at least $x \%$ of the volume; $V_{x \%}$, volume receiving at least $x \%$ of the dose; $V_{y G y}$, volume receiving at least $y$ Gy dose. Statistical significance (Fisher's sign): a=electrons vs RA_1, b=electrons vs RA_2, c=RA_1 vs RA_2.

The planning time was about $40 \mathrm{~min}$ for RA and about 30 min for electrons on a stand-alone Eclipse system running on a Dell T5500 station (Dell Inc., Round Rock, TX) with $20 \mathrm{~GB}$ of random-access memory and eight cores. RA planning was therefore a factor of 1.3 longer than electrons.

The delivery time for these plans were as follows: 12.1 \pm

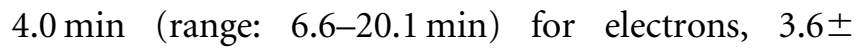
$1.0 \mathrm{~min}$ (range: $1.9-4.7 \mathrm{~min}$ ) for RA_1 and $4.8 \pm 1.3 \mathrm{~min}$ (range: 3.0-6.7 $\mathrm{min}$ ) for RA_2. RA delivery was therefore a factor 2.5-3.4 faster than electrons. Electron plans were delivered with the fastest dose rate- $600 \mathrm{MU}$ per minute. The difference between delivery times for electrons and RA is statistically significant, as is it significant between RA_1 and RA_2.

\section{DISCUSSION}

The radiation treatment of cutaneous KS is frequently performed with usage of electron beams. The present study investigated at planning level the possibility to adopt VMAT with RA for these patients. The primary objective of the study was to demonstrate the possibility to adequately cover the target. Statistically significant improvement was achieved with respect to electrons. The secondary objective was the possibility to spare healthy tissues inside the leg. This is achievable with electrons but not, for example, with conventional photon beams with three-dimensional conformal or with opposed fields. VMAT demonstrates this possibility (RA_2 option) when appropriate planning objectives are adopted, without clinically meaningful deterioration of target coverage. Therefore, having proven that VMAT is dosimetrically advantageous, the study addressed the question of practical delivery aspects. From the point of view of the MU, i.e. the intrinsic efficiency, both techniques were similar (electrons and RA_1), while RA_2 was less efficient. This is justified by the fact that, to generate a "dosimetric hole" surrounded by a thin layer of uniform dose, a high degree modulation is required, and this correlates with the number of MUs. 
Planning time did not result in particularly macroscopic differences. On one side, the need to define several electron fields with proper alignments and matching leads to a substantial equivalent time consumption as the relatively fast optimisation of RA plans with the $\mathrm{PRO} 3$ algorithm [16]. On the other side, AcurosXB calculation of RA plans is as fast as the eMC (electron dose calculation would be longer if generalised gaussian models would be used). In addition, planning efficiency would depend on the total number of planning stations available in the clinic. More relevant is, from a practical point of view, the huge difference in delivery time observed between the two techniques. In this case, RA delivery was a factor of 2.5-3.4 faster than electrons. The treatment efficiency issue is particular important when incidence of KS is large or growing fast. The situation in South Africa could be paradigmatic; Sitas and Newton [20] showed how in less than 10 years, between 1988 and 1996, incidence of KS raised by a factor of $>3$ owing to the HIV epidemics. Some areas of South Africa, like KwaZuluNatal, are at the epicenter of the HIV pandemic and, given the very limited access to antiretroviral therapy, there are large numbers of HIV-related KS treated in the public sector needing adequate and highly efficient radiation therapy. Globally, in such areas where incidence of KS is increasing and resources are limited, VMAT RA treatment could constitute an important improvement in the management of this disease.

A problem not directly addressed in the study is the immobilisation of patients. This can be easily managed by means of several commercial or home-made devices (e.g. vacuum cushions for RA). RA allows for easier solutions since it does not require irradiation of patients from the contralateral side, while with electrons this might be necessary and the need of direct electron fields from the base of the feet is definitely a practical complication. In practice, the need to irradiate the leg from $360^{\circ}$, avoiding irradiation of the contralateral leg, would require quite a lot of effort in developing adequate positioning tools. In addition, motion management (leg movement owing to patient's discomfort) is more relevant for the slower technique, electrons, than for RA.

To conclude, two issues remain unaddressed in this study: (i) availability of CT to scan patients and (ii) availability of VMAT RA. These issues might constitute some limiting factors given that not all linear accelerators are equipped for VMAT while availability of electrons is more widely spread, and access to CT for a patient's scan could be limited. The present study does not pretend to solve these kinds of issues but aims to be seminal in the determination of the most effective strategies to attack a health problem. To consolidate the feasibility study presented in this report, a clinical Phase II trial has been designed to start treatment of patients affected by this pathology. In addition, the treatment technique described here can be adopted in a broader group of patients when similar target volumes should be treated. An example of this extended application could be the treatment of any kind of superficial or skin tumours in the elderly (important also in more wealthy areas like Europe or North America) or in the young; in both cases, for different reasons, minimisation of bone irradiation could be fundamental.

\section{CONCLUSION}

The present study showed that VMAT RA could be considered as a valuable tool for radiation treatment of cutaneous KS. Results showed that (i) target homogeneity can be largely improved compared with electrons; (ii) sparing of internal leg can be further improved; and (iii) treatment time can be reduced to $<5$ min compared with $\sim 12$ min with electrons. Busy clinics in environments with limited resources, with high incidence of KS (especially the epidemic form) could strongly benefit from the adoption of this modality of treatment. A clinical Phase II protocol is ongoing to prove the clinical impact.

\section{CONFLICT OF INTEREST}

LC acts as Scientific Advisor to Varian Medical Systems and is Head of Research and Technological Development at the Oncology Institute of Southern Switzerland, Bellinzona.

\section{REFERENCES}

1. Aldenhoven M, Barlo NP, Sanders CJ. Therapeutic strategies for epidemic Kaposi's sarcoma. Int J STD AIDS 2006;17:571-8.
2. Cooper JS, Steinfeld AD, Lerch I. Intentions and outcomes in the radiotherapeutic management of epidemic Kaposi's sarcoma. Int J
Radiat Oncol Biol Phys 1991;20: 419-22.

3. Nobler MP, Leddy ME, Huh SH. The impact of palliative irradiation 
on the management of patients with acquired immune deficiency syndrome. J Clin Oncol 1987;5:107-12.

4. Singh NB, Lakier RH, Donde B. Hypofractionated radiation therapy in the treatment of epidemic Kaposi sarcoma-a prospective randomized trial. Radiother Oncol 2008;88: 211-16.

5. Stein ME, Lachter J, Spencer D, Margolius L, Bezwoda WR. Variants of Kaposi's sarcoma in Southern Africa. A retrospective analysis (1980-1992). Acta Oncol 1996;35:193-9.

6. Berson AM, Quivey JM, Harris JW, Wara WM. Radiation therapy for AIDS-related Kaposi's Sarcoma. Int J Radiat Oncol Biol Phys 1990;19: 569-75.

7. Harrison M, Harrington KJ, Tomlinson DR, Stewart JS. Response and cosmetic outcome of two fractionation regimens for AIDS-related Kaposi's sarcoma. Radiother Oncol 1998;46:23-8.

8. Conill C, Alsina M, Verger E, Henríquez I. Radiation therapy in AIDS-related cutaneous Kaposi's sarcoma. Dermatology 1997;195: 40-2.

9. Geara F, Le Bourgeois JP, Piedbois P, Pavlovitch JM, Mazeron JJ. Radiotherapy in the management of cutaneous epidemic Kaposi's sarcoma. Int J Radiat Oncol Biol Phys 1991;21:1517-22.

10. Otto K. Volumetric modulated arc therapy: IMRT in a single arc. Med Phys 2008;35:310-17.

11. Llacer-Moscardo C, Quenet F, Azria D, Fenoglietto P. Feasibility study of volumetric modulated arc therapy for the treatment of retroperitoneal sarcomas. Radiat Oncol 2010;5:83.

12. Fogliata A, Scorsetti M, Navarria P, Catalano M, Clivio A, Cozzi L, et al. Dosimetric comparison between VMAT with different dose calculation algorithms and protons for soft tissue sarcoma radiotherapy. Acta Oncol 2012; in press. Epub ahead of print Jun 2012.

13. Zacarias A, Brown M, Mills M. Volumetric modulated Arc therapy (VMAT) treatment planning for superficial tumors. Med Dosim 2010;35:226-9.

14. International Commission on Radiation Units and Measurements. ICRU Report 83. Prescribing recording and reporting photon beam intensity modulated radiation therapy (IMRT). Oxford, UK: ICRU Oxford University Press; 2010.

15. Fogliata A, Bergstroem S, Cafaro I, Clivio A, Cozzi L, Dipasquale G, et al. Cranio spinal irradiation with volumetric modulated arc therapy: a multi institutional treatment experience. Radiother Oncol 2011;99: 79-85

16. Vanetti E, Nicolini G, Nord J, Peltola J, Clivio A, Fogliata A, Cozzi L. On the role of the optimization algorithm of RapidArc ( $\left.{ }^{\circledR}\right)$ volumetric modulated arc therapy on plan quality and efficiency. Med Phys 2011;38: 5844-56.

17. Vassiliev O, Wareing T, McGhee J, Failla G, Salehpour M, Mourtada F. Validation of a new grid based Blotzmann equation solver for dose calculation in radiotherapy with photon beams. Phys Med Biol 2010; 55:581-98.

18. Neuenschwander H, Mackie TR, Reckwerdt PJ. MMC-a high performance Monte Carlo code for electron beam treatment planning. Phys Med Biol 1994;40: 543-74.

19. Janssen J, Korevaar E, Battum L, Storchi P, Huizenga H. A model to determine the initial phase space of a clinical electron beam from measured beam data. Phys Med Biol 2001;46:269-86.

20. Sitas F, Newton R. Kaposi's sarcoma in South Africa. J Natl Cancer Inst Monogr 2001;28:1-4. 